\title{
Antibody disulfide bond reduction during process development: Insights using a scale-down model process
}

\author{
Julie Ruaudel", Martin Bertschinger, Sonia Letestu, Roberto Giovannini, Paul Wassmann, Pierre Moretti \\ From 24th European Society for Animal Cell Technology (ESACT) Meeting: C2P2: Cells, Culture, Patients, Products \\ Barcelona, Spain. 31 May - 3 June 2015
}

\section{Background}

Keywords:mAb reduction / disulfide bond scrambling / free thiols

During the development of the production process for a monoclonal antibody (mAb), we observed a significant increase in the reduction of interchain disulfide bonds following pilot scale protein A purification of the produced antibody $(\operatorname{IgG} 1 \kappa)$. Different companies have reported the presence of fragmented IgG1 antibodies in the clarified cell culture fluid (CCCF) at manufacturing scale. It has been demonstrated [1] that enzymes of the thioredoxin system, released during the decline phase of the culture, were responsible for the interchain disulfide bond reduction. However, our observations were different as the majority of the interchain disulfide bonds were still oxidized in the CCCF. The massive fragmentation of interchain disulfide bonds occurred only after pilot scale protein A purification step. This study presents our insights in antibody reduction using scale-down models.

\section{Experimental approach}

First, the mAb fragmentation phenomenon was reproduced at small scale by incubation of mAb containing CCCF with cell lysate followed by SDS-PAGE with and without NEM (N-ethylmaleimide). NEM is known to protect free thiol groups during sample preparation for SDS-PAGE [2].

Second, a scale down model of the bioreactor process was established in order to understand which process parameters led to the massive reduction and hence fragmentation of the $\mathrm{mAb}$ during the pilot scale protein $\mathrm{A}$ purification process.

* Correspondence: Julie.Ruaudel@glenmarkpharma.com Glenmark Pharmaceuticals, La Chaux-de-Fonds, Switzerland

\section{Results and discussion}

This study demonstrated first that the massive increase in $\mathrm{mAb}$ fragmentation could be replicated experimentally in small-scale when the antibody was subjected to protein denaturing conditions. Fully oxidized antibodies were observed in the CCCF using non-reducing SDS PAGE with NEM (protecting the free thiol groups), whereas without NEM, the interchain bonds of the antibodies were massively reduced. We concluded from these experiments that intradomain free thiols can reduce interchain disulfide bonds by disulfide scrambling when these are exposed to denaturing conditions during SDS-PAGE preparation or protein A elution at pilot scale. We also demonstrated that the accumulation of free thiols in the supernatant, observed at the start of the decline phase, led to the increase of free intradomainthiols in the mAb present in the CCCF at the end of the culture. In parallel, the intracellular ratio GSH/ GSSG showed a sharp increase from day 7 onwards, indicating a change in the intracellular redox potential in the process. One hypothesis is that these accumulated intracellular reductive forms were released in the supernatant as soon as the viability decreased, leading to a more reductive environment. The detailed mechanisms responsible for this intracellular change remain unclear. Figure 1 summarizes our understandings of the phenomenon.

\section{Conclusion}

MAb reduction is a complex mechanism, which was found in our case to be related to the upstream process rather than a specific cell line. We observed this phenomenon with two different IgG1 $\kappa$ expressing $\mathrm{CHO}$ cell lines in two different non-optimized processes, in which the cell viability decreased quickly and sharply. As this 


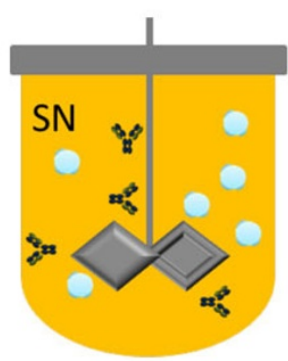

Early culture: No reduction

- Antibody production

- High viability

- Intracellular thiol accumulation

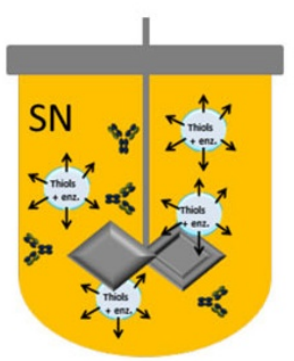

Mature culture: Reduction of disulfide bridges in $\mathrm{mAb}$ (intradomain + interchain) Viability drops:

- Enzyme release into SN

- Release of free thiols into SN

- Shift of redox potential in SN

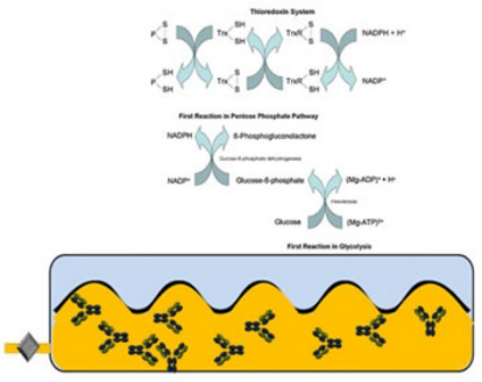

CCCF: Antibodies with reduced intradomain and interchain disulfide bonds.

During clarification viability drops further:

- Enzyme release (thioredoxin,...)

- Release of free thiols into SN

- Shift of redox potential in SN

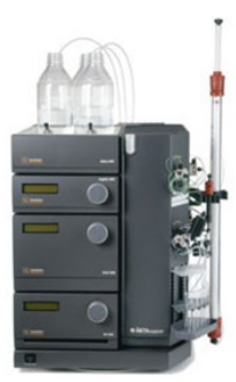

Post protein A: Disulfide scrambling (minimum scale) leads to reduction of interchain bonds: Fragmented antibodies
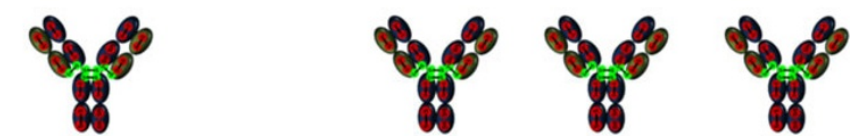

Increasing reduction of intradomain and interchain disulfide bonds

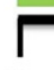

Inoculation
Mature culture

\section{Harvest + Clarification}

Figure 1 Our current understanding of the process leading to $\mathrm{mAb}$ reduction during pilot scale protein $\mathrm{A}$ purification

viability decline was identified to be the root cause for the release of thiols in the supernatant, the most evident way to prevent the reduction phenomenon might be the modification of the process conditions in order to maintain a good viability throughout the entire upstream process until the clarification step. Further, different IgG formats have been shown to have different sensitivities to reduction [3], thus choosing the right IgG format might be helpful to avoid reduction. The addition of copper sulfate has been also identified as a treatment in the literature [4]. Added at the beginning of the culture, copper sulfate maintains the supernatant in an oxidative state until the end of the culture and thus prevents an increase of free thiols in the supernatant.

\section{Acknowledgments}

All Glenmark teams involved in this project (CLD, USP, DSP, QC, Immunology and AE)
Prevention of Antibody Disulfide Bond Reduction During Cell Culture Manufacturing. Biotechnology and Bioengineering 2010, 106(3).

2. Hongcheng Liu, Georgeen Gaza-Bulseco, Chris Chumsae, Abigail NewbyKew: Characterization of lower molecular weight artefact bands of recombinant monoclonal IgG1 antibodies on non-reducing SDS-PAGE. Biotechnol Lett 2007, 29:1611-1622.

3. Hutterer KM, Hong RW, Lull J, Zhao X, Wang T, Pei R, Le ME, Borisov O, Piper R, Liu YD, Petty K, Apostol I, Flynn GC: Monoclonal antibody disulfide reduction during manufacturing: Untangling process effects from product effects. MAbs 2013, 5(4):608-13, Jul-Aug;: doi: 10.4161/ mabs.24725. Epub 2013 Apr 18.

4. Wendy BChaderjian, Edward TChin, Reed JHarris, Tina MEtcheverry: Effect of copper sulfate on performance of a serum-free $\mathrm{CHO}$ cell culture process and the level of free thiol in the recombinant antibody expressed. Biotechnol Prog 2005, 21:550-553.

\section{doi:10.1186/1753-6561-9-S9-P24}

Cite this article as: Ruaudel et al: Antibody disulfide bond reduction during process development: Insights using a scale-down model process. BMC Proceedings 2015 9(Suppl 9):P24.

\section{Published: 14 December 2015}

\section{References}

1. Melody Trexler-Schmidt, Sandy Sargis, Jason Chiu, Stefanie Sze-Khoo,

Melissa Mun, Yung-Hsiang Kao, Michael WLaird: Identification and 